\title{
Status of the Solid Xenon Project at Fermilab
}

\author{
R. B. Pahlka ${ }^{a *}$ \\ ${ }^{a}$ Fermi National Accelerator Laboratory, \\ P. O. Box 500, Batavia, IL 60510, USA \\ E-mail: pahlka@fnal.gov
}

\begin{abstract}
The solid (crystalline) phase of xenon possesses many of the same advantages of liquid xenon as a particle detector material including good transparency and ionization drift, selfshielding, low intrinsic background, and high scintillation light yield. Many of the properties of solid xenon have been measured previously employing small volumes and thin films. However, few systematic studies have been successfully produced using large volumes of solid xenon. Two major $\mathrm{R} \& \mathrm{D}$ issues must be addressed to make a solid xenon particle detector; the demonstration of the scalability of solid xenon and the capability to readout solid xenon signals. Both issues are being addressed with a dedicated cryogenic system at Fermilab. The first phase of this project entailed growing approximately a kilogram of transparent solid phase xenon and was successfully completed in 2010 at Fermilab. The second phase of this project is underway where the signals from scintillation light and electron drift in solid xenon will be measured. These measurements are expected to be completed this year. In this talk, we will discuss the recent progress of solid xenon detector R\&D performed at Fermilab.
\end{abstract}

KEYWORDS: liquid xenon; solid xenon; scintillation.

${ }^{*}$ R. B. Pahlka 


\section{Contents}

1. Introduction 囵

2. The Cryogenic Facility 2

3. Solid Xenon Growth

4. The Devices [3

5. Preliminary Results

6. Future Plans

\section{Introduction}

Particle detection using the liquid and gaseous phases of xenon is well-established by experiments currently in operation [1, [ [ ] ]. The properties of xenon as a detection medium make it a good choice in many applications. Xenon posesses no long-lived radioisotopes which would lead to intrisic backgrounds. The high scintillation light yield, combined with a peak scintillation wavelength of $175 \mathrm{~nm}$ in a range of good optical transparency, can provide calorimetric measurements with standard photon detection devices. Xenon has a relatively high melting point at $161 \mathrm{~K}$ which facilitates simple cryogenic designs and is easy to purify.

Frozen (solid) xenon in crystalline form shows high potential for particle detection as a new technology when deployed in large volumes. Solid xenon posesses all the qualities of liquid xenon, but with many distinct advantages. In crystal form, the face centered cubic crystal structre is simple to exploit. Solid xenon posesses an even larger scintillation light yield compared to the liquid phase. Further background contamination is mitigated, which makes it ideal for low background experiments. Solid xenon exhibits a faster electron drift velocity compared to liquid xenon. Finally, at milliKelvin temperatures, the phonon channel can be an important additional source of information.

Many potential applications have been considered including low background counting experiments such as solar axion searches, dark matter searches, and searches for neutrinoless double beta decay with enriched ${ }^{136} \mathrm{Xe}$. Additional applications include solar neutrino measurements with depleted ${ }^{136} \mathrm{Xe}$, supernova detection, neutrino coherent scattering, as well as applications in medicine. In these proceedings, we report on the status of R\&D being conducted at The Fermi National Accelerator Laboratory on the characterization of the particle detection properties of solid xenon. This work evolved out of a New Initiative R\&D project under the Fermilab Center for Particle Astrophysics with the goal to demonstrate that approximately $1 \mathrm{~kg}$ of optically transparent solid xenon 

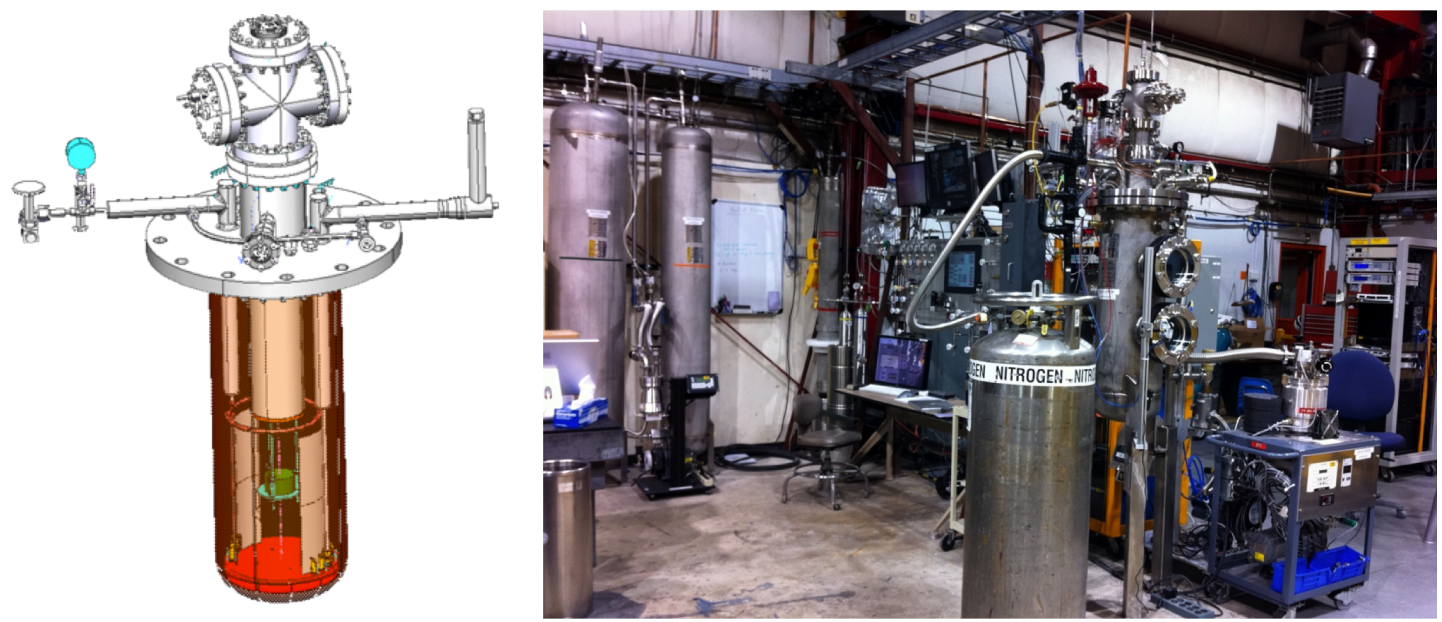

Figure 1. Left: A drawing of the cryogenic vessel. The innner glass xenon vessel is surrounded by a second glass vessel. This is surrounded by a vacuum space, all contained in a steel capsule.

could be produced. The system was completed in September 2011 and was operational in December 2011. The first system tests were performed in January 2012 and the Phase One goal was achieved in mid-2012. The second phase of the project, now underway, is to automate the growth process and the cryogenics, further refine the growth parameters, and measure the scintillation light output and electron drift in solid xenon. We will discuss the cryogenic facility, previous success in solid xenon production and growth, the devices installed in the vessel, and our future plans. We will focus principally on the scintillation light readout from solid xenon.

\section{The Cryogenic Facility}

All devices are controlled under a gas monitoring system developed at Fermilab with system safety locks installed on the critical pressure regulators. This system controls the liquid nitrogen level using electronic valves and a barometer which correlates the temperature and pressure to the height of liquid nitrogen in the vessel. The height of liquid nitrogen, combined with a resistive heater wound around the inner glass vessel, subsequently controls the temperature of the gaseous and liquid xenon. Employed in conjunction with varying the pressure of the inner glass chamber, gaseous xenon is first condensed to liquid, then as applicable, carefully frozen under monitored and controlled conditions.

Figure 11 shows the configuration of the cryogenic vessel and the system setup at Fermilab. The cryogenic vessel contains two smaller glass vessels and consists of a steel cylinder with three glass viewports. A 4 inch diameter inner glass vessel houses the xenon and other devices. Surrounding this is a 9 inch diameter outer glass vessel. These are then surrounded by the vacuum-jacketed steel vessel. The figure also shows the various sub-systems, pumps, gas control, and xenon storage.

The xenon transfer from the storage facility is controlled by a pressure differential between the xenon tank and the inner glass vessel, under the "cryogenic pump" concept. The outer glass vessel is filled with a small amount of liquid nitrogen facilitating the pressure differential. The xenon passes through a noble gas purifier and a universal gas analyzer before reaching the vessel. 

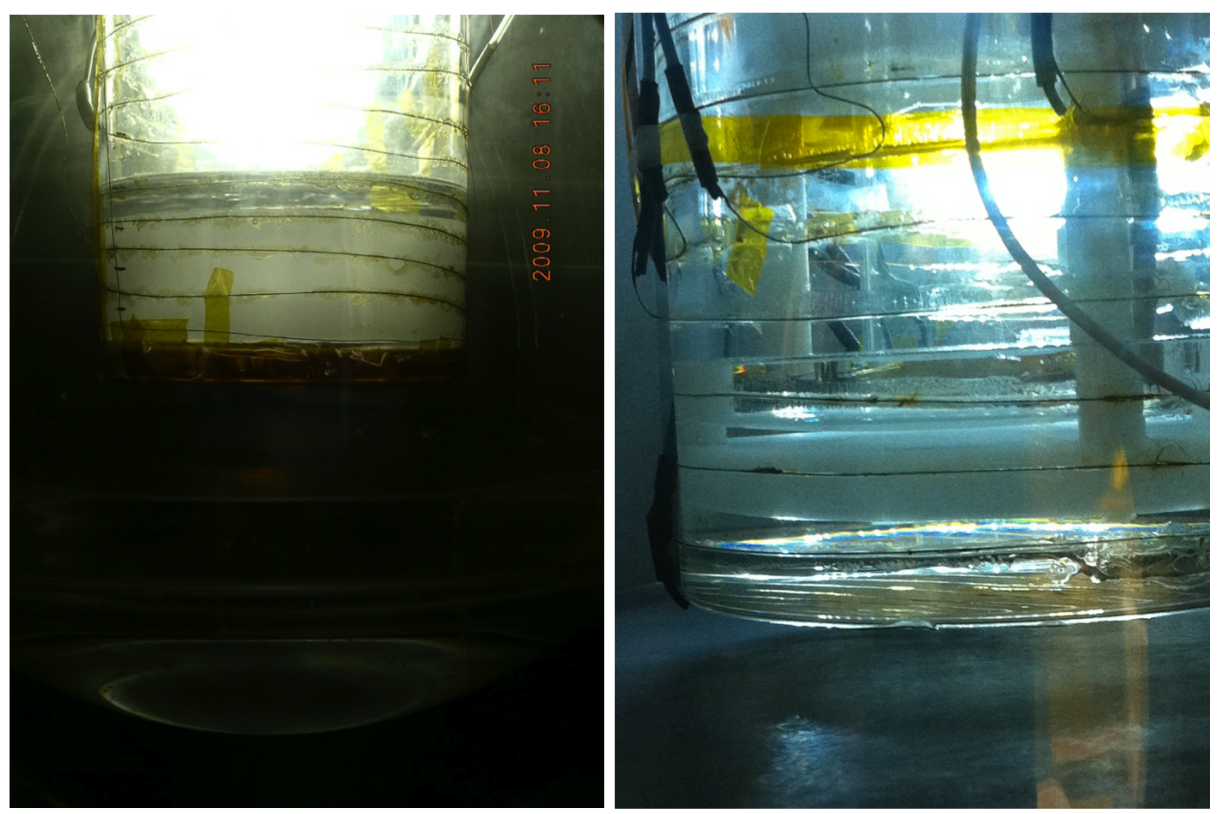

Figure 2. Left: Solid xenon growth under sub-optimal conditions producing poor transparency. Right: Solid xenon growth under good conditions. Several distinct boundaries exist starting with transparent frozen xenon, then a larger layer of liquid. The white disk near the bottom is part of the teflon support structure.

The purification system can be configured to allow multiple passes of the xenon gas through the purifier en route to the chamber. The transfer of xenon to the chamber has recently been improved with a new liquid nitrogen transfer line, and is now greatly simplified.

\section{Solid Xenon Growth}

The Phase One goal was to demonstrate the growth of approximately $1 \mathrm{~kg}$ of optically transparent solid xenon. This was achieved with an established recipe as follows. Gaseous xenon was first condensed to liquid inside the vessel until $90 \%$ of the available xenon was transferred. Seven resistive thermal devices (RTD), along with resistive heaters placed on the outside of the vessel, monitored and controlled the temperature. The temperature of the top-most RTD was set to $160 \pm 0.5 \mathrm{~K}$. The temperature of the bottom-most RTD was set to $145 \pm 0.5 \mathrm{~K}$. The xenon gas pressure at the top of the liquid was set to $1.0 \pm 0.1 \mathrm{~atm}$. With these settings, the growth rate of optically transparent solid xenon was about $3 \mathrm{~cm}$ in 10 hours. We found that solid xenon could be grown faster by simply reducing the temperature, but this resulted in poor optical transparency, characteristic of the amorphous, non-crystal form. Figure 2 shows the results of two independent growth trials indicating a marked difference between how the solid xenon was produced.

\section{The Devices}

In these proceedings, we focus on describing the capability of the chamber setup to collect and measure scintillation light from liquid and solid xenon. We have constructed a small calorimeter using two photomulitiplier tubes (PMTs) to measure the scintillation light from ionizing radiation. 


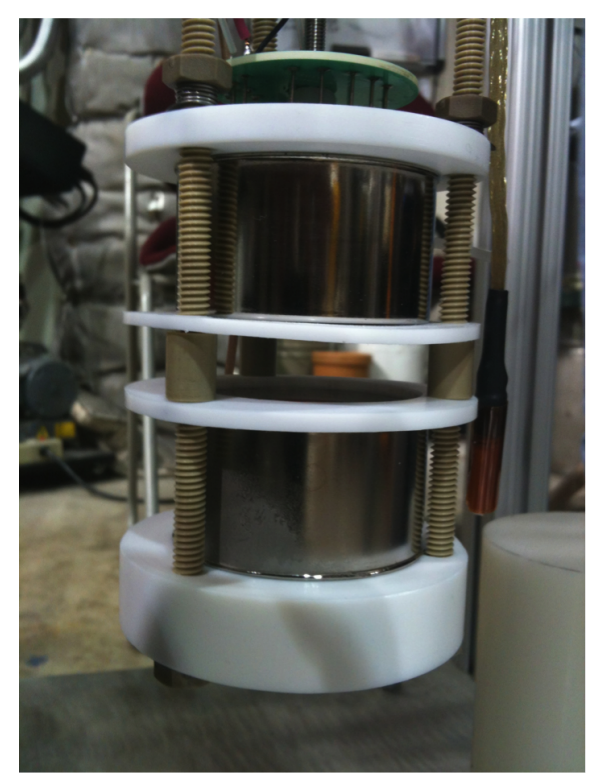

Figure 3. A photo of the PMT configuration installed in the inner glass vessel. The teflon rings provide mechanical support and alignment. The deposited energy from cosmic rays or radioactive sources is measured within the gap separating the two PMTs.

The PMTs are 2 inch diameter Hamamatsu R6041-406MOD high quantum efficiency cyrogenic tubes which have a good response to the $175 \mathrm{~nm}$ scintillation light from xenon. In our preliminary setup, the tubes are oriented facing each other, separated by a small gap, in which liquid or solid xenon is introduced. Between the tubes is a cylindrical ring made of acrylic with a reflective mylar layer to enhance the light collection.

We employ a single-mode fiber coupled to a light emitting diode (LED) pulsed with a highfrequency function generator to create light to characterize the single photoelectron spectra which is used for calibration. The light from the fiber is injected into the space between the PMTs through a small opening in the acrylic ring. A data acquisition system from CAEN, with a suite of versatile software developed on-site, is used to collect data and store individual waveforms directly into ROOT files. A small window in the reflector, with a mirror oriented to reflect light into the region between the PMTs, facilitates the calibration technique. For data acquisition using cosmic rays or radioactive sources, we measure the deposited energy in units of ADC counts and correlate this to the number of photons collected based on the ADC value of the single photoelectron at a specified voltage. Figure 3 shows the configuration of the PMTs employed in the setup. This setup is flexible and allows for varying the separation of the tubes, providing valuable cross-checks and comparative measurements.

\section{Preliminary Results}

The PMT setup described above facilitates the measurement of the energy spectra from radioactive isotope sources. The setup allows for the introduction, placement, and measurement of pin sources or disk sources. Several improvements to the PMT setup have recently been made and the design is evolving quickly. Figure $₫$ shows the results from an exposure of solid xenon to a ${ }^{207} \mathrm{Bi}$ source in 


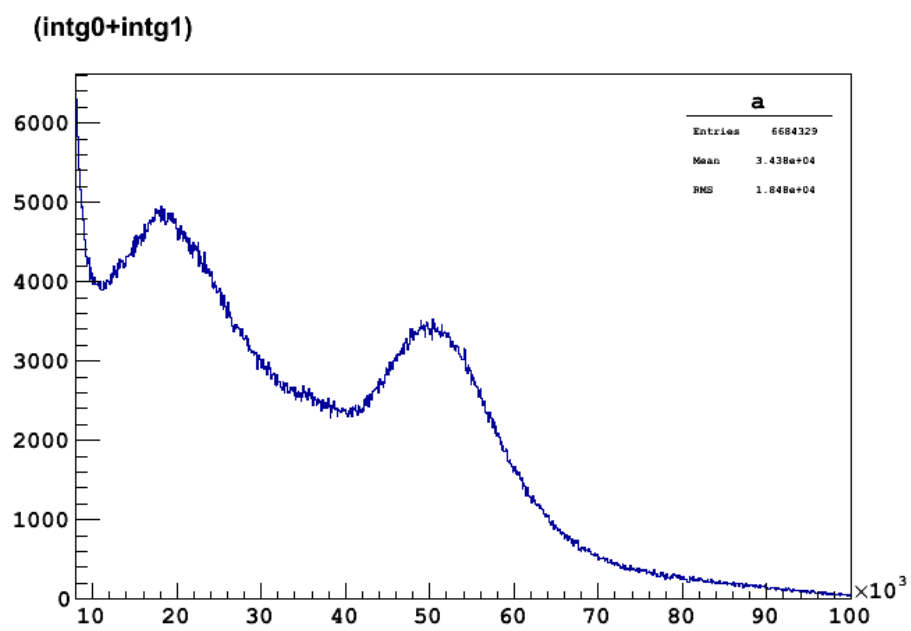

Figure 4. The energy spectra of ${ }^{207} \mathrm{Bi}$ measured by scintillation light from solid xenon registered in the two PMTs in units of ADC counts. The coincidence signals from both PMTs are summed using the digitized pulse waveforms. The temperature of the vessel was $145 \mathrm{~K}$.

units of ADC counts. This isotope has several conversion electron peaks, with the two lowest (and most intense) at approximately 481 and $976 \mathrm{keV}$. The two conversion electron peaks are clearly present, confirming the ability to measure scintillation light from solid xenon. Work is in progress to convert the units to single photoelectron charge once the calibration procedure is finalized.

\section{Future Plans}

We have reported on the status of the solid xenon project at Fermilab. We have demonstrated that the crystalline phase of solid xenon can be produced quickly and reliably and that the growth is controlled under carefully monitored conditions. We have demonstrated that solid xenon emits scintillation light and we are in the process of refining our measurements to compare the properties of solid and liquid xenon scintillation. We will characterize the scintillation light yield with several radioactive sources. We will also characterize and measure the electron drift lifetime and electron drift velocity in solid xenon and compare the results to liquid. We will also install a Timepix tracking module to determine the spatial resolution of charged particle tracks.

\section{Acknowledgments}

This work was supported by the U.S. Department of Energy. We are grateful to the Fermilab technical staff for providing assistance with system construction and cryogenics.

\section{References}

[1] M. Auger et al. [EXO Collaboration], Phys. Rev. Lett. 109, 032505 (2012)

[2] N. Ackerman et al. [EXO-200 Collaboration], Phys. Rev. Lett. 107, 212501 (2011)

[3] A. Minamino [XMASS Collaboration], Nucl. Instrum. Meth. A 623, 448 (2010) 